biomarkers as predictors for postoperative aspiration pneumonia and in-hospital mortality.

Results Mean age of patients was $81.4 \pm 8.6$ (SD) years old. 26 patients (14\%) developed aspiration pneumonia and 19 patients (10\%) died before discharge. Preoperative biomarkers/ parameters: Body mass index (BMI) $18.5 \pm 9.2 \mathrm{~kg} / \mathrm{m}^{2}$, Serum albumin (Alb) $2.8 \pm 0.5 \mathrm{~g} / \mathrm{dL}$, BUN $22.8 \pm 16.7 \mathrm{mg} / \mathrm{dL}$, Total lymphocyte count (TLC) $1498 \pm 744 / \mu L$, Onodera's Prognostic Nutritional Index (O-PNI) 35.7 \pm 6.8 , Geriatric Nutritional Risk Index (GNRI) 76.7 \pm 18.6. AUC (Area under the curve) for the outcome of aspiration pneumonia after PEG: BMI 0.593 (95\%CI: $0.490-0.695)$, Alb 0.656 (95\%CI: $0.567-$ $0.746)$, BUN 0.676 (95\%CI: 0.565-0.787), TLC $0.612(95 \%$ CI: $0.507-0.717$ ), O-PNI 0.687 (95\%CI: 0.585-0.789), GNRI 0.683 (95\%CI: $0.580-0.787$ ). AUC for postoperative in-hospital mortality: BMI 0.626 (95\%CI: 0.482-0.770), Alb 0.642 (95\%CI: $0.528-0.757)$, BUN 0.835 (95\%CI: 0.750-0.921), TLC 0.652 (95\%CI: $0.525-0.778)$, O-PNI 0.685 (95\%CI: 0.576-0.794), GNRI 0.680 (95\%CI: 0.557-0.804). A cut-off value of $24.5 \mathrm{mg} / \mathrm{dL}$ yielded a sensitivity of $78.9 \%$ and a specificity of $78.4 \%$ for in-hospital mortality.

Conclusions Higher preoperative BUN levels predict aspiration pneumonia after PEG reasonably well and is a superior prognostic factor for in-hospital mortality when compared to other traditionally used biomarkers or even widely used risk indices such as O-PNI and GNRI. As BUN is also a frequently measured biomarker, clinicians should take it into account during preoperative assessment of patients undergoing PEG.

\section{IDDF2018-ABS-0187 JEJUNAL TUMOUR - A PARADIGM SHIFT FROM OPEN TO MINIMALLY INVASIVE APPROACH}

Lai Fen Wong*. University Malaya Medical Centre, Malaysia

\subsection{6/gutjnl-2018-IDDFabstracts. 148}

Background Jejunal tumour is a rare, uncommon tumour of the gastrointestinal (GI) tract. The symptoms and signs are vague, non-specific and frequently confused with other GI manifestations, for instance, GI bleed or obstruction. Due to its rarity, we are facing diagnostic dilemma and variation in management jejuna tumour.

Methods We are reporting a case series of five patients with jejunal tumours with various presentations, for instance, obscure gastrointestinal bleed (OGIB), by far the commonest presentation follows by obstruction and abdominal pain. Presentation of OGIB and diagnosed via capsule endoscopy whereby ulcer was seen at jejunum in two patients and tumour was seen in one patient. Two patients diagnosed with jejunal tumours via contrasted CT abdomen with the presentation of abdominal pain and distension post-prandial in one patient and intestinal obstruction another patient.

Push enteroscopy was used as diagnostic tool in all patients except in patient with obstruction and biopsy of lesions/ulcers taken for histology examination.

Subsequently, all patients underwent laparoscopic segmental small bowel resection and intracoporeal primary anastomosis.

Results The average operating time was 2.3 hours with the length of stay of 3.33 days. Patients were allow orally 24 hours postoperatively. The analgesia requirement was minimum. There were no significant complications recorded, i.e. no postoperative ileus and surgical site infection.
Conclusions Traditionally, a patient with jejunal tumour often being diagnosed during laparotomy and intraoperative enteroscopy for overt GI bleed. In this modern era of medicine, we are going towards a minimally invasive approach in term of patient's management. Push enteroscopy should be considered in cases of overt GI bleed whereby gastroscopy and colonoscopy revealed normal findings. It's a diagnostic tool as well as an adjunct tool to tattoo for laparoscopic jejunal resection and intracoporeal primary anastomosis.

Push enteroscopy expedites the diagnosis and management of jejunal tumour.

Minimally invasive surgery has a minimal interruption of the patient's physiology which offers quicker recovery after surgery as compared to open surgery.

\section{IDDF2018-ABS-0188 EFFECT OF NURSING INTERVENTION ON ACETIC ACID CHROMOENDOSCOPY FOR DETERMINING CHRONIC ATROPHIC GASTRITIS}

Wenhui Tan*, Jiachuan Wu, Fang Wang, Lifang Ye, Fang Lv, Ling He, Qian Kang. Guangdong Second Provincial General Hospital, China

\subsection{6/gutjnl-2018-IDDFabstracts. 149}

Background To investigate the effect of the nursing intervention on acetic acid chromoendoscopy (AAC) for determining chronic atrophic gastritis.

Methods 60 patients with chronic atrophic gastritis were enrolled in our study from January 2016 to August 2017. Patients were randomly divided into AAC group $(n=30)$ and non-AAC group $(n=30)$. After endoscopic management and nursing intervention, SAS and SDS scores, clinical characteristics, individual tolerance and endoscopic diagnosis were collected and analysed both groups.

Results No statistical significance between ACC group and non-ACC group in SAS and SDS scores, clinical characteristics and individual tolerance $(\mathrm{p}>0.05)$. DBP, SBP and pulse were much lower in ACC group than non-ACC group $((\mathrm{p}>0.05))$. The accuracy, sensitivity and specificity were 91.33\%,76.82\% and $97.38 \%$ respectively in the ACC group, while they were $86.24 \%, 75.26 \%$ and $95.41 \%$ in the non-ACC group.

Conclusions Acetic acid chromoendoscopy is valuable for the diagnosis of chronic atrophic gastritis. And nursing intervention could safely and vastly improve SAS and SDS scores, DBP, SBP and pulse, even the tolerance during gastroscopy.

\section{IDDF2018-ABS-0192 THE ASSOCIATION BETWEEN GASTRIC POLYPS AND COLORECTAL NEOPLASIA: A LARGE PROSPECTIVE CROSS-SECTIONAL STUDY}

Shenghong Zhang*, Danping Zheng, Shanshan Huang, Li Li, Minhu Chen. Department of Gastroenterology, The First Affiliated Hospital, Sun Yat-sen University, Guangzhou, China

\subsection{6/gutjnl-2018-IDDFabstracts. 150}

Background Today, it is not clarified whether patients with gastric polyps without any alarming symptoms for colorectal neoplasia need colonoscopy screening. The objective of this study is to prospectively determine the association between gastric polyps and colorectal neoplasia. 